\title{
A Rare Cause of Airway Collapse: Spontaneous Hemorrhage and Rupture of a Parathyroid Adenoma
}

Fergal G. Kavanagh, MB BCh BAO, BMed Sc (Hons), MRCSI, FCAI, FJFIMI'; Shane Brennan, MB BCh BAO, MRCPI, DipRCPath²; Paul Lennon, MB BCh, MD, MSc, FRCS (ORL-HNS)1

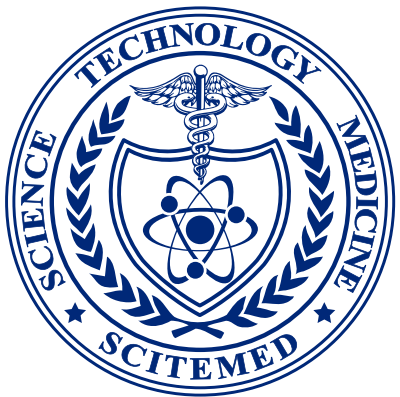

'Department of Otolaryngology Head and Neck Surgery, St James's Hospital, Dublin 8, Ireland

${ }^{2}$ Department of Histopathology and Cytopathology, St James's Hospital, Dublin 8, Ireland

\begin{abstract}
Parathyroid adenomas are a benign neoplastic process that account for $80-85 \%$ of primary hyperparathyroidism and typical clinical presentation is associated with signs and symptoms pertaining to or complications arising from hypercalcemia. This case-based review will focus on an atypical presentation of a parathyroid adenoma. This is the case a fifty-two-year-old female who presented to the Emergency Department with a three-day history of left side neck swelling, odynophagia, and cervical ecchymosis. Her condition deteriorated rapidly while in the Emergency Department. She developed stridor and airway compromise necessitating endotracheal intubation. Computed Tomography of the neck revealed an extensive soft tissue attenuation throughout the left neck with a $3.5 \mathrm{~cm}$ rounded mass arising from the inferior pole of the left thyroid gland resulting in right sided tracheal deviation. Initial biochemical investigations were normal. Intraoperatively, posterior to the left thyroid gland, a large hemorrhagic mass was identified and excised. Histopathological examination confirmed a parathyroid adenoma within which was extensive hemorrhage, with capsular distortion. In conclusion, this is a rare case presentation of a parathyroid adenoma with airway collapse and normal biochemistry due to hemorrhage and rupture of a parathyroid adenoma.
\end{abstract}

\section{INTRODUCTION}

Parathyroid adenomas are the most common cause of hyperparathyroidism, with a prevalence of $0.2-0.5 \%$ [1]. Patients classically present with signs and symptoms pertaining to and/or complications arising from hypercalcemia including osteoporosis and renal calculi. This case-based review will focus on atypical presentations of a parathyroid adenoma.

The first documented report illustrating a parathyroid adenoma with extracapsular hemorrhage was in 1934 [2]. From the limited number of case reports published to date, presentation is classically with signs of extensive cervical ecchymosis in the region, as well as signs and symptoms secondary to compression of adjacent structures within the neck $[3,4]$. These cases are challenging to diagnosis pre-operatively as they occur abruptly, without a known precipitant and are infrequent. A further anomaly of this case relates to the biochemical markers being within the normal range; the mechanism by which this can occur will be explained.

This is the first published case report of a patient presenting with stridor from an acute, near complete, airway obstruction with normal biochemical markers, as the initial presentation of a parathyroid adenoma, which required immediate surgical intervention.

\section{CASE REPORT}

A fifty-two-year-old female presented with a 3-day history of odynophagia, sore throat and anterior neck pain radiating into her mediastinum. Over the preceding 24 hours she developed localised left sided neck swelling with intensifying pain and 'noisy breathing'. She presented to the Emergency Department with diffuse neck swelling and ecchymosis. The ecchymosis was more prominent on the left side with associated tenderness. She had no history of neck trauma. She had no past medical or surgical history. She was not taking any medications or anticoagulants, and she had no known allergies.
Initial investigations conducted included biochemical full blood count and coagulation tests were within normal limits including calcium. A parathyroid hormone (PTH) level was not carried out. Radiological investigations included initial neck and chest $x$-ray studies which were unremarkable. Her condition deteriorated and she developed stridor with respiratory distress necessitating an emergency endo-tracheal intubation.

An urgent computed tomography (CT) neck which demonstrated extensive soft tissue attenuation throughout the left neck and left lobe of the thyroid gland, with right sided tracheal deviation. There was a $3.5 \mathrm{~cm}$ spherical

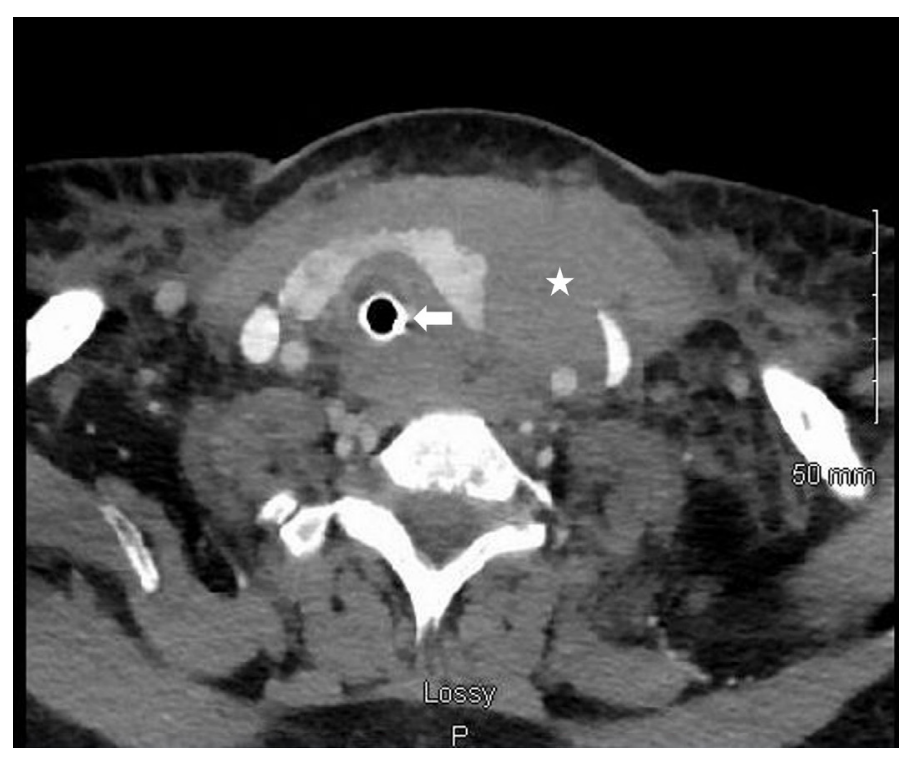

Figure 1. Axial image of neck computed tomography demonstrating extensive soft tissue attenuation (asterisk) within the left neck causing marked mass effect and tracheal deviation to the right (arrow). 


\section{CASE REPORT}

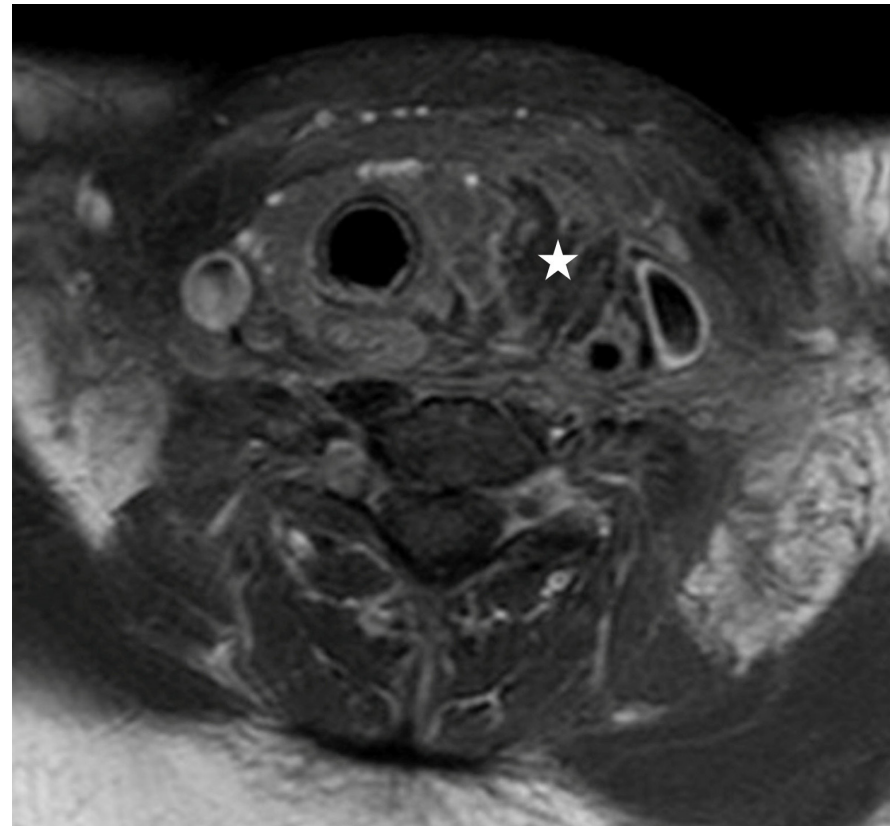

Figure 2. Post-contrast axial magnetic resonance image of the neck demonstrating a well circumscribed, walled off, $3.2 \mathrm{~cm}$ left paratracheal neck mass (asterisk) inferior to the left lobe of the thyroid.

mass arising from the inferior pole of the left thyroid gland (Figure 1). Extensive fat stranding into the anterior mediastinum was also identified. A magnetic resonance image (MRI) neck to further clarify these findings demonstrated a $3.2 \mathrm{~cm}$ paratracheal neck mass inferior to the lower pole of the left thyroid gland with surrounding soft tissue oedema (Figure 2).

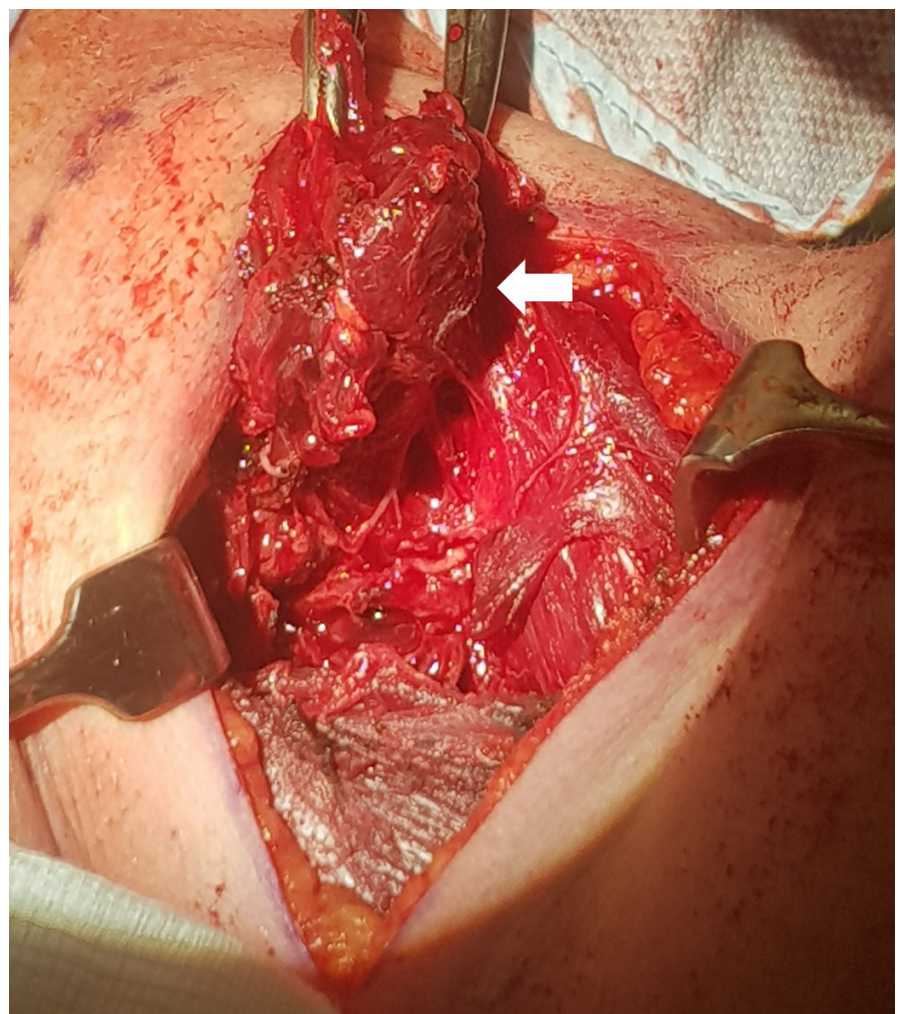

Figure 3. Clinical intraoperative photograph showing friable thyroid gland and tissue (arrow).

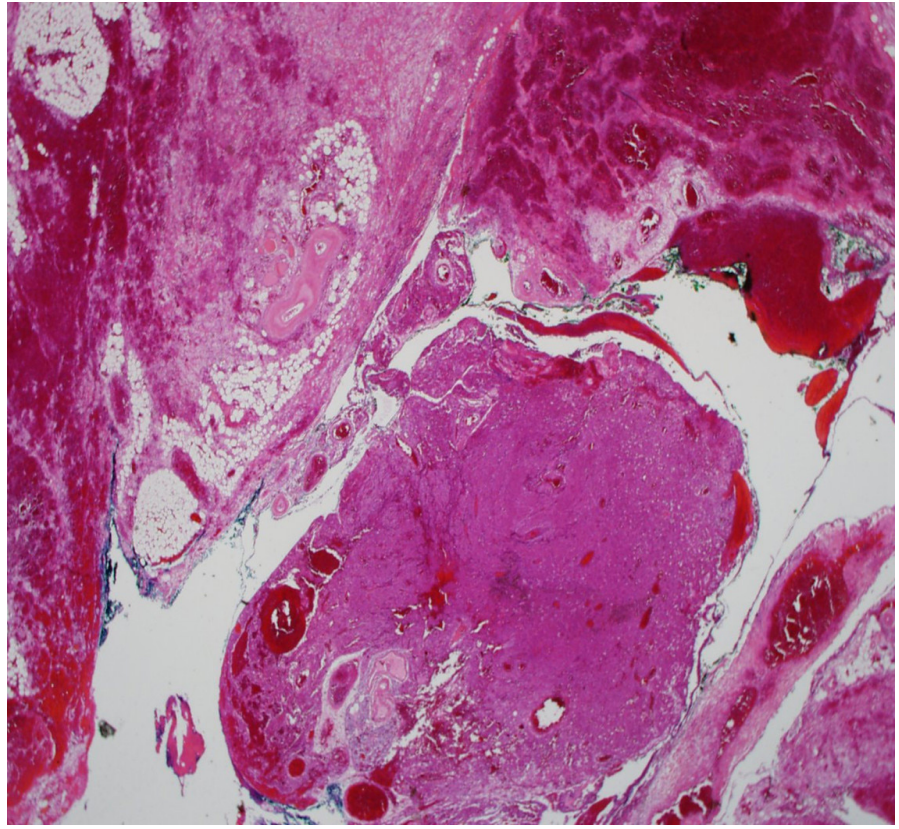

Figure 4. Low power magnification showing central sheets of parathyroid chief cells with surrounding hemorrhage and fat necrosis.

A decision was made for urgent surgical intervention which consisted of hematoma evacuation and 'en bloc' left thyroid lobectomy including left parathyroidectomy. Intra-operatively the neck was found to be swollen due to the hematoma; no abscess was detected (Figure 3). A fragmented mass was present posterior to the left thyroid gland lobe. Notable oozing was identified in this area without any active bleeding. Vascular structures were intact.

The specimen was sent for histopathological assessment. On gross examination, the specimen was composed of hemorrhagic material surrounding the left lobe of the thyroid gland. Piecemeal grey tissue was present, but no distinctive mass. Histological examination on Hematoxylin and Eosinophil (H\&E) staining showed fragmented tissue containing benign thyroid glandular structure with extensive perithyroidal hemorrhage and fat necrosis. There was an enlarged cellular parathyroid structure present with showing marked hemorrhage, thrombus formation and necrosis, consistent with a parathyroid adenoma. The appearances microscopically in correlation with the surgical findings were that of hemorrhage and rupture of a parathyroid adenoma (Figure 4).

Post-surgical management included a bedside laryngoscopy confirmed the absence of anatomical airway abnormalities that could have contributed to her presentation. Further hematological evaluation out-ruled any underlying coagulopathy. Extubation in the critical care unit was uneventful, and she was transferred to the ward after 24 hours and made a full recovery.

Follow up serological biochemical calcium and PTH levels were within normal limits. The patient was reviewed in outpatients shortly post discharge, and at 6-months, there were no outstanding surgical or medical issues identified.

\section{DISCUSSION}

Parathyroid adenomas usually come to clinical attention due to the combined effects of increased PTH secretion or hypercalcemia. These are known as 'bones, stones, abdominal moans and psychic groans' [5]. This article focuses on atypical presentations of this condition.

Rupture of parathyroid adenoma and consequent hemorrhage in the neck is exceedingly rare. Spontaneous neck hemorrhage is a rare surgical emergency. Apart from trauma the most common causes are vessel rupture 
or spontaneous extra-glandular bleeding from the thyroid or parathyroid gland. A review of the case presentations in the literature to date proposed a clinical triad consisting to acute neck swelling, hypercalcemia and neck/chest ecchymosis point to this clinical hypothesis [6,7]. A variety of less reported clinical presentations include neck pain, dysphagia, dysphonia and chest pain and shortness of breath from mediastinal tracking of the hemorrhage have been reported [8].

In terms of the primary pathology the accepted theory to explain hemorrhage of parathyroid adenomas is that they develop as a result of the rapid growth, overwhelming their vascular supply [8]. Rupture of the adenoma is deemed more likely than intra/extra thyroidal hemorrhage, due to the thin nature of the parathyroid capsule. Given the posterior location of the parathyroid glands, patients are more likely to present with dysphagia and chest pain from tracking of the hemorrhagic/necrotic material into the mediastinum. Bleeding into a thyroid cyst and subacute thyroiditis can produce similar symptoms and should be considered in the initial evaluation, bearing in mind that neither condition is necessarily associated with hypercalcemia. Anticoagulation or bleeding diathesis may be a predisposing factor in such cases [9]. Mediastinal parathyroid adenoma account for $1.4 \%$ of all parathyroid adenomas. Thus, mediastinal parathyroid hemorrhage is exceedingly rare; mimicking an aortic dissection and acute circulatory collapse [10].

There are a number of unique aspects to this case. This is the first published care in the literature of an acute airway collapse secondary to the parathyroid adenoma. The provided CT images show the degree of both deviation and narrowing of the airway (Figure 1). Given the clinical context of rapid airway compromise, the images are post endotracheal intubation. Despite this, the severity of the airway compression can still be appreciated.

Particular to our case was the acuity in which the patient needed definite surgical management. The optimal timing for removal of the hemorrhagic and necrosed adenoma remains controversial. Regarding the published case reports in the literature, time at which surgical excision was performed varied from weeks to months post presentation and Chaffanion et al. had proposed performing surgery at 3 months allowing easier dissection and reducing complication rate [11]. However, as occurrences within the literature are limited, there is no optimal time period elucidated, and thus the clinical context must be taken into consideration. In our case we proceeded with surgery as there was already airway compromise and the diagnosis was only made retrospectively.

A further challenging aspect of this presentation was that the patient presented with normal calcium and PTH levels. Spontaneous remission of primary hyperparathyroidism due to auto-infarction of parathyroid adenoma is a phenomenon also known as 'parathyroid autoinfarction' [12]. Rapid growth of the parathyroid adenoma leads to cystic degeneration or acute tissue necrosis from the compressive effect of the surrounding hematoma. Post operatively calcium and PTH must be monitored as ischemic adenomatous tissue has the potential to re-grow leading to the recurrence. This explains the reason this patient presented with normal biochemistry and in her case there was no re-growth of tissue with normal calcium and PTH observed at her outpatient follow up.

\section{CONCLUSION}

The learning point from this case is to ensure a broad differential is placed forward when dealing with acute neck swelling. Routine Head and Neck pathology can present in an unusual fashion, with resultant challenging presentations. To the best of our knowledge this is the first report of a presentation with spontaneous hemorrhage and rupture of a parathyroid adenoma with airway compromise.

\section{ARTICLE INFORMATION}

*Correspondence: Fergal G. Kavanagh, MB BCh BAO, BMed Sc (Hons), MRCSI, FCAI, FJFIMI
Department of Otolaryngology Head and Neck Surgery, St James's Hospital, James Street, Dublin 8, Ireland. Email: fergalkavanagh@rcsi.ie

Received: Mar. 12, 2021; Accepted:Jul. 10, 2021; Published: Oct. 25, 2021

\section{DOI: 10.24983/scitemed.aohns.2021.00147}

Disclosure: This study was presented and won first prize at the Royal Academy of Medicine in Ireland on Dec. 06, 2020, held in the Royal Victoria Eye and Ear Hospital, Adelaide Road, Dublin 2.

Author Contributions: FK wrote the initial manuscript. SB and PL contributed to review and editing of the manuscript. All authors approved the final manuscript.

Ethics Approval and Consent to Participate: The study is in accordance with the ethical standards of the 1964 Helsinki declaration and its later amendments or comparable ethical standards. Full informed consent was obtained from the patient for the publication of this case-based review.

Funding: No sources of funding from research funding bodies was sought.

Conflict of Interest: The authors declare that this case report was conducted in the absence of any commercial or financial relationships that could be construed as a potential conflict of interest.

Copyright @ 2021 The Author(s). This is an open-access article distributed under the terms of the Creative Commons Attribution 4.0 International License (CC-BY).

\section{REFERENCES}

1. Christensson T, Hellstrom K, Wengle B, Alveryd A, Wikland B. Prevalence of hypercalcaemia in a health screening in Stockholm. Acta Med Scand 1976;200(12):131-137.

2. Capps RB. Multiple parathyroid tumors with massive mediastinal and subcutaneous hemorrhage: A case report. Am J Med Sci 1934;188:800-805.

3. Deveze A, Sebag F, Pili S, Henry JF. Parathyroid adenoma disclosed by a massive cervical hematoma. Otolaryngol Head Neck Surg 2006;134(4):710-712.

4. Ulrich L, Knee G, Todd C. Spontaneous cervical haemorrhage of a parathyroid adenoma. Endocrinol Diabetes Metab Case Rep 2015;2015:150034.

5. Bilezikian JP, Brandi ML, Rubin M, Silverberg SJ. Primary hyperparathyroidism: New concepts in clinical, densitometric and biochemical features. J Intern Med 2005;257(1):6-17.

6. Kozlow W, Demeure MJ, Welniak LM, Shaker JL. Acute extracapsular parathyroid hemorrhage: Case report and review of the literature. Endocr Pract 2001;7(1):32 36.

7. Simcic KJ, McDermott MT, Crawford GJ, Marx WH, Ownbey JL, Kidd GS. Massive extracapsular hemorrhage from a parathyroid cyst. Arch Surg 1989;124(11):1347-1350

8. Yoshimura $\mathrm{N}$, Mukaida $\mathrm{H}$, Mimura T, et al. A case of an acute cervicomediastinal hematoma secondary to the spontaneous rupture of a parathyroid adenoma. Ann Thorac Cardiovasc Surg 2014;20 Suppl:816-820.

9. Taniguchi I, Maeda T, Morimoto K, Miyasaka S, Suda T, Yamaga T. Spontaneous retropharyngeal hematoma of a parathyroid cyst: Report of a case. Surg Today 2003;33(5):354-357.

10. Santos GH, Tseng CL, Frater RW. Ruptured intrathoracic parathyroid adenoma. Chest 1975;68(6):844-846.

11. Chaffanjon PC, Chavanis N, Chabre O, Brichon PY. Extracapsular hematoma of the parathyroid glands. World J Surg 2003;27(1):14-17.

12. Norris EH. Primary hyperparathyroidism; a report of five cases that exemplify special features of this disease (infarction of a parathyroid adenoma; oxyphil adenoma). Arch Pathol (Chic) 1946;42:261-273. 